Supporting Information for

\title{
Structure of Electric Double Layer under Cationic Langmuir Monolayer: Charge Condensation Effect
}

\author{
Sona Krem, Minho Lee, Sokhuoy Sam, Woongmo Sung, and Doseok Kim* \\ Department of Physics, Sogang University, Seoul, Korea
}

\section{Experiments}

II. Time series SFVS spectra

III. Integrated potential using a MPB solution

IV. Condensed layer in the MPB model 


\section{Experiments}

1-hexadecanol (HD, Sigma Aldrich) and 1,2-dipalmitoyl-3-trimethylammonium-propane (DPTAP, Sigma Aldrich) were dissolved in chloroform solution and 1:10 (v:v) methanol-chloroform mixture, respectively, to prepare $1 \mathrm{mg} / \mathrm{ml}$ stock solutions. Then HD:DPTAP mixtures (0:1, 1:1, 2:1, 4:1, 8:1, 16:1, 32:1, and 1:0 molar ratios) were obtained by mixing appropriate amount of the above stock solutions. These mixture solutions were then spread on petri dishes with different salt concentrations for SFVS measurements. After spreading the solutions on the subphase surface, at least 10 minutes were allowed for chloroform evaporation and monolayer stabilization. In the SFVS measurements, mean surface area/alkyl chain of the HD:DPTAP mixtures monolayers were $22.5 \AA^{2} /$ chain and the spectrum were taken at room temperature $\left(20^{\circ} \mathrm{C}\right)$. Previous simulation and experimental studies showed that the monolayers consisting of different types of phospholipids and fatty amine are homogeneously mixed, especially when the headgroup of one of the constituents is charged. ${ }^{1,2}$ For this reason, it is believed that the miscibility at the molecular level exists in the mixture monolayer in this study. $\mathrm{NaCl}(99.999 \%$ trace metals basis, Sigma-Aldrich) salt were dissolved in ultrapure water (conductivity higher than $18.2 \mathrm{M} \Omega . \mathrm{cm}$ ). pH 5.7 water solution were obtained from air-equilibrated ( 14 hours) distilled water.

Femtosecond, broadband SFVS setup consisted of Ti:Sapphire amplifier (LIBRA-HE, Coherent, 2.5 mJ/pulse, 40 fs pulse duration, $1 \mathrm{kHz}$ repetition rate, center wavelength $\sim 800 \mathrm{~nm}$ ), of which $1 \mathrm{~mJ}$ of the upstream pulse pumped the commercial optical parametric amplification (OPA) and noncollinear difference frequency generation (NDFG) systems (Light Conversion, TOPAS-Prime) for mid-IR pulse generation (bandwidth $250 \mathrm{~cm}^{-1}$ ). The output DFG pulse (mid-IR) energy was $\sim 6 \mu \mathrm{J}$ at the sample stage. The remaining $1.5 \mathrm{~mJ}$ of the upstream pulse were introduced to a Fabry-Perot etalon to narrow the bandwidth of the visible to about $15 \mathrm{~cm}^{-1}$ FWHM. The energy of the visible was about $25 \mu \mathrm{J}$ at the sample stage. The broadband IR and narrowband visible pulses overlapped at the sample surface, and four different IR center wavelengths (2720 nm, $2850 \mathrm{~nm}, 3150 \mathrm{~nm}$, and $3400 \mathrm{~nm})$ were used to fully cover $\mathrm{CH}$ and $\mathrm{OH}$ regions. Polarizations of the detected sum-frequency, visible, and IR pulses were S, S, and P, respectively. Intensified CCD camera (iStar 320T, Andor Technology, thermoelectrically cooled to $-30{ }^{\circ} \mathrm{C}$ ) mounted on a monochromator (Shamrock 303i, Andor) was used as a detector of the sum-frequency signal. The sum-frequency spectra of the mixtures were collected for 30 seconds for each IR wavelength setting. The SFVS spectra were normalized against the broadband infrared beam profile using nonresonant SFVS spectra from a z-cut quartz crystal. 


\section{Time series SFVS spectra}

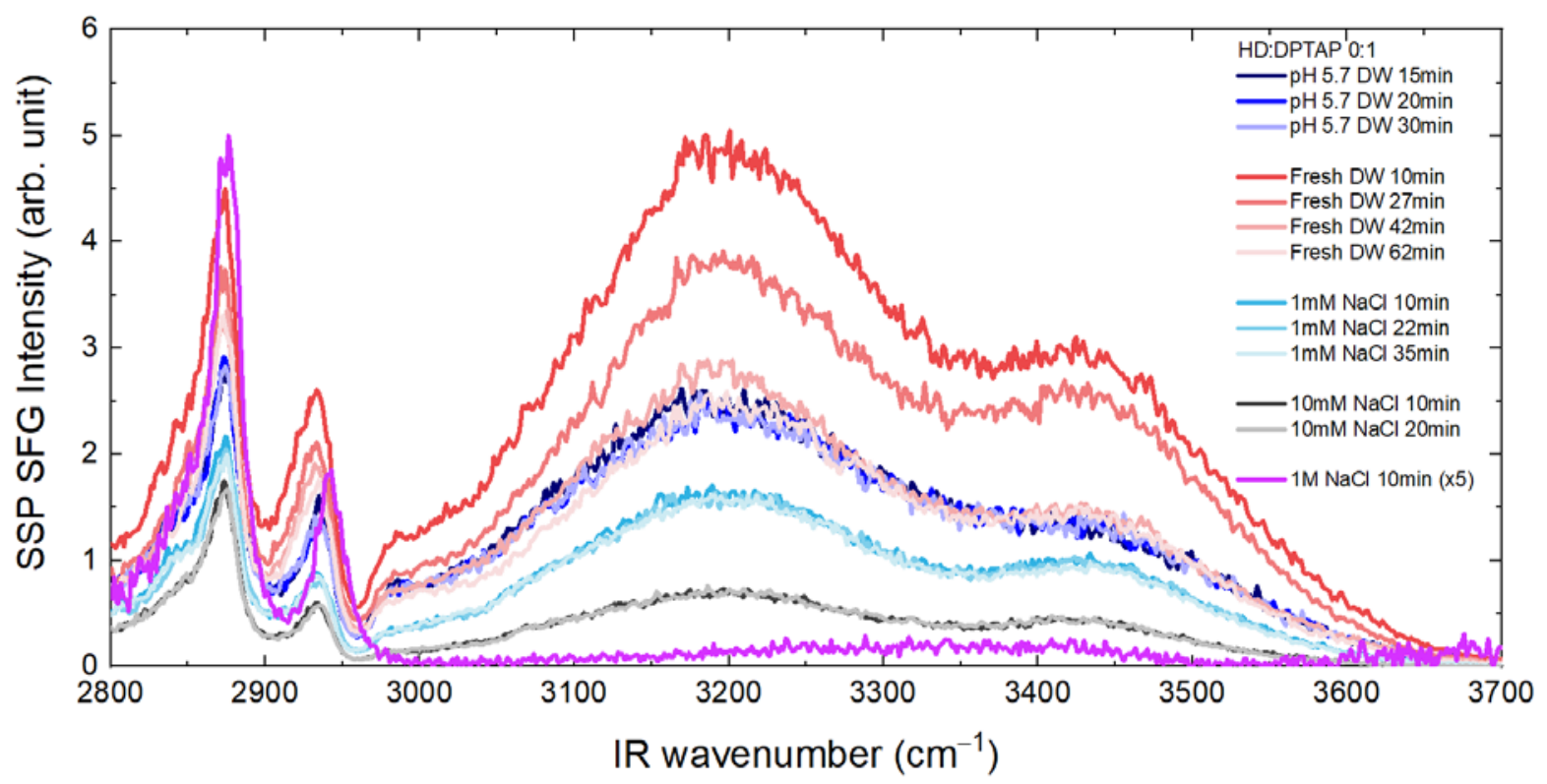

Figure S1. Time-series sum frequency spectra from DPTAP Langmuir monolayer (HD:DPTAP = 0:1) at different ionic strengths: the light red lines are from fresh distilled water (DW), the light blue lines are from pH 5.7 distilled water air-equilibrated for $14 \mathrm{~h}$, the light cyan lines are from $1 \mathrm{mM} \mathrm{NaCl}$ solution, the grey lines are from $10 \mathrm{mM}$ $\mathrm{NaCl}$ solution, and the magenta line (5x magnified) is from $1 \mathrm{M} \mathrm{NaCl}$ solution. The time was counted just after spreading the monolayer in the petri dish.

$\mathrm{CO}_{2}$ in air dissolves in distilled water and lowers $\mathrm{pH}$ from 7 to 5.7. Due to change in ionic strength, SFG spectra of pure DPTAP monolayer on fresh distilled water (DW) (light red lines in Fig. S1) monotonically decreased with time. To overcome such difficulties, the distilled water had been allowed to air-equilibrate for 14 hours with the final pH 5.7. Evidently, SFG spectra (light blue lines in Fig. S1) of pure DPTAP monolayer on this air-equilibrated water showed no time dependence. By contrast, the spectra from DPTAP on the subphase solutions of $1 \mathrm{mM}$ and 10mM NaCl (light cyan and grey lines in Fig. S1, respectively) hardly changed with time. SFG spectrum from DPTAP monolayer with $1 \mathrm{M} \mathrm{NaCl}$ subphase is shown (magenta line, $5 \mathrm{x}$ magnified) to give semiquantitative estimate of $\chi_{\mathrm{s}}^{(2)}$. 


\section{Integrated potential using a MPB solution}
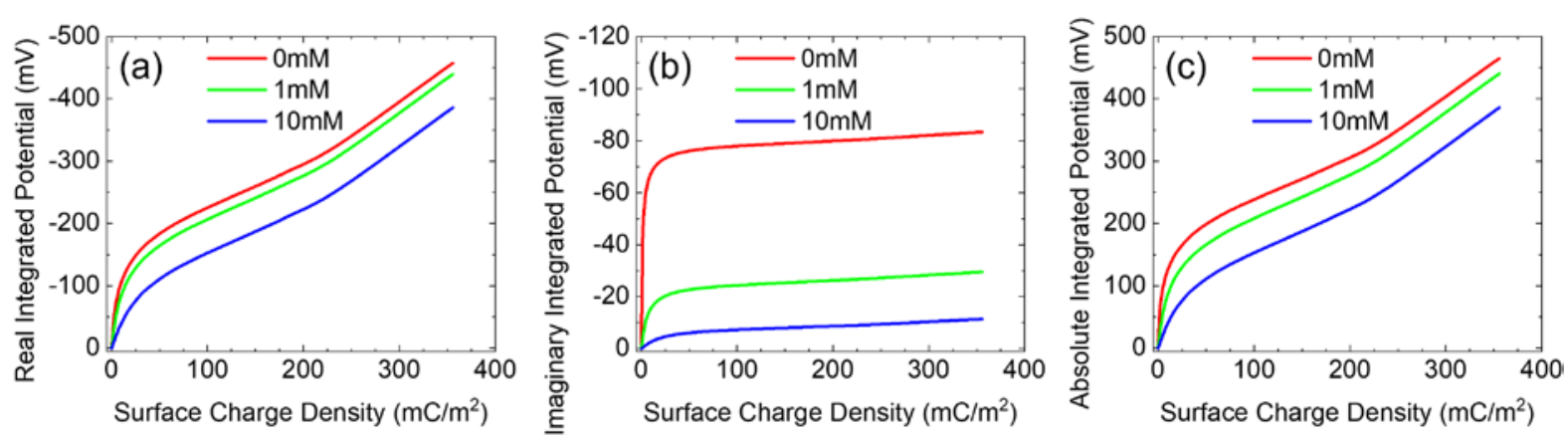

Figure S2. Integrated potential $\Psi=-\int_{z_{0}}^{\infty} \frac{d \phi(z)}{d z} \exp \left(i \Delta k_{z} z\right) d z$ calculated by the GC theory (MPB solution): (a) Real part, (b) Imaginary part, and (c) absolute values of $\Psi$ versus the surface charge density for different added salt concentrations. $\phi(z)$ was calculated from numerical solution of Eq. (7) shown in the main text. The wavevector mismatch $\Delta k_{z}$ in our beam geometry is about $1 /(45 \mathrm{~nm})$ and the size of the hydrated counterion is 7.5 Å.

\section{Condensed layer in the MPB model}

In the MPB model, the thickness of condensed layer could also be estimated to be ${ }^{3} l_{c, M P B}=a^{3} \sigma / e . a, \sigma$, and $e$ are the size of the hydrated counterion, surface charge density, and the elementary charge, respectively. MPB model (black line in Fig. S3) predicts that the condensed layer is formed even at very low surface charge density. This unphysical outcome is due to an approximation used in defining the condensed layer. ${ }^{3}$ On the other hand, the CDL model separates the diffused layer from the condensed layer (calculated from Eqs. (9) and (10) of the paper) when it begins to form due to steric limit. The condensed layer (red, green, and blue lines) is formed only above specific surface charge density, which makes it clear to determine critical surface charge density. 


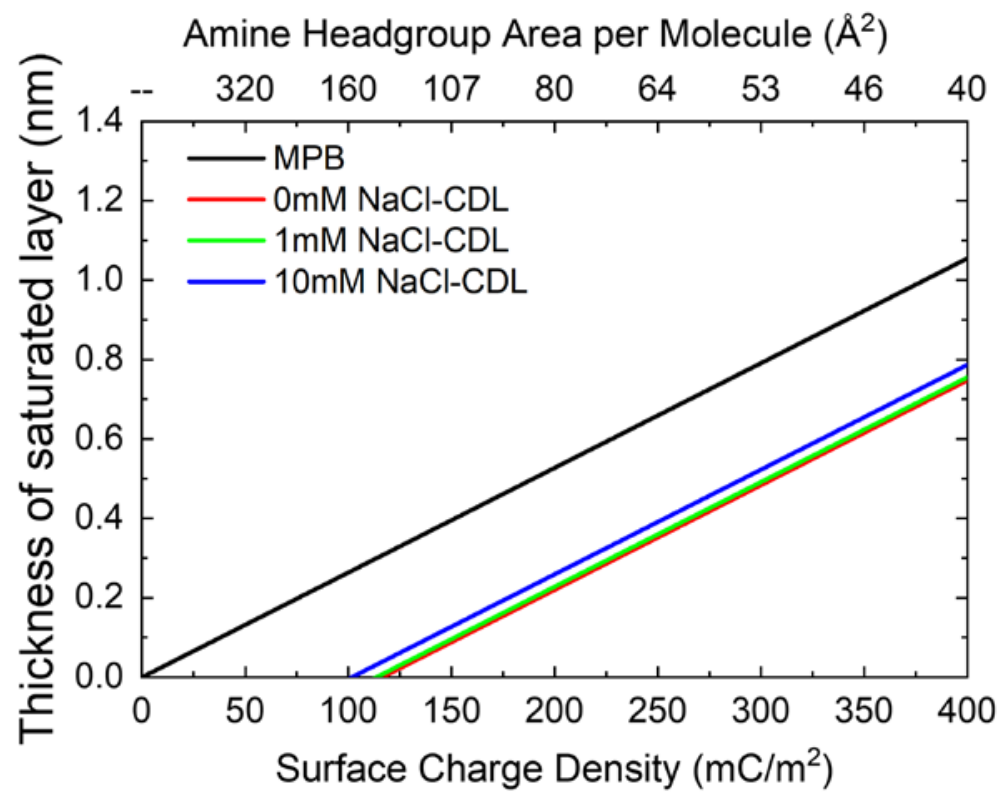

Figure S3. Comparison of thickness of condensed layer predicted by MPB model and CDL model. The size of the hydrated counterion is $7.5 \AA$.
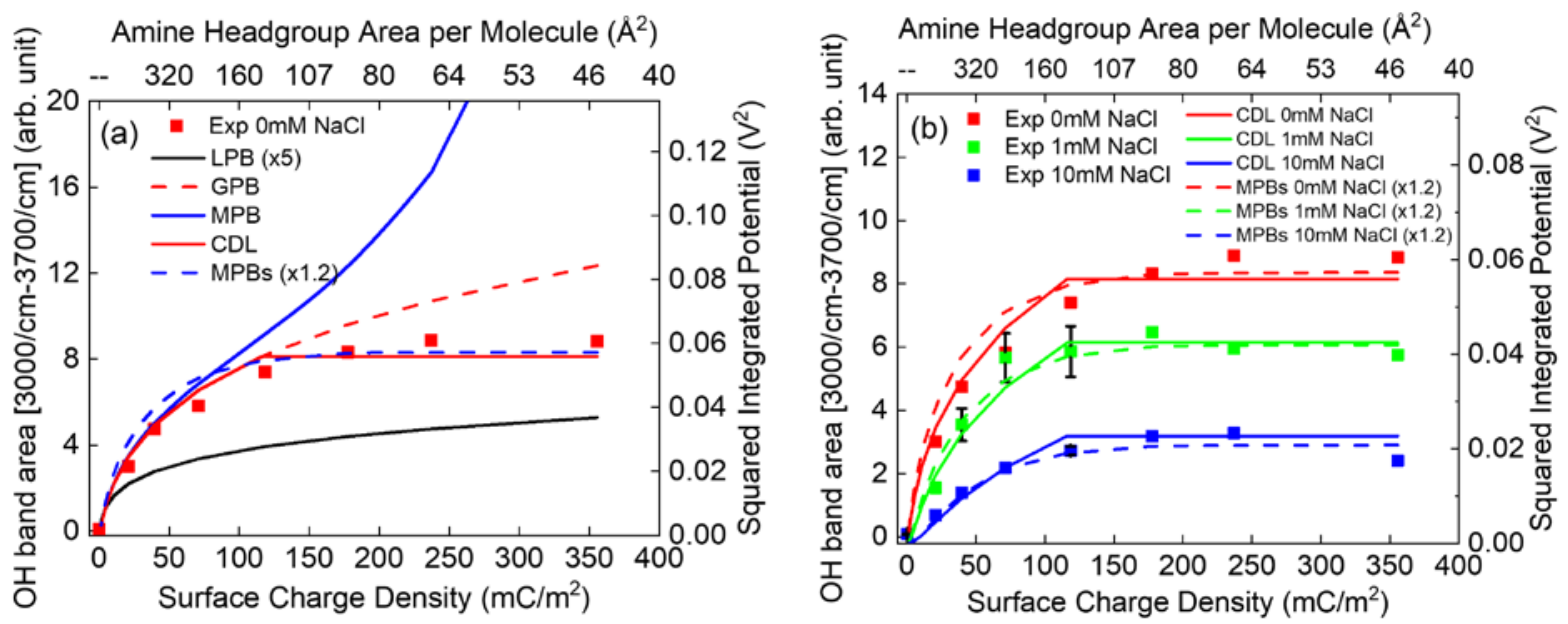

Figure S4. (a). Integrated areas of the $\mathrm{OH}$ bands from Fig. 2(a) in the main text (red squares) vs. square of the integrated potential $|\Psi|^{2}$ obtained from the theory (LPB, GPB, MPB, and CDL). The dashed blue line (termed MPBs) is $|\Psi|^{2}$ with the lower limit $\left(z_{0}\right)$ in the integral in Eq. (2) set to $l_{c, M P B}$, calculated in Fig. S3. (b). Integrated areas of the $\mathrm{OH}$ bands at different subphase salt concentrations (from Fig. 2 in the main text) vs. $|\Psi|^{2}$ from the CDL and MPBs. 
Figure S4 compares the $|\Psi|^{2}$ from various models in Fig. 2, with an addition of $|\Psi|^{2}$ calculated from MPB model with an exclusion of condensed layer (termed MPBs in Fig. S4, integration of Eq. (2) started from $l_{c, M P B}$ ). The predicted $\mathrm{OH}$ band areas are almost the same between these two models, and both followed the experimental data fairly well. The MPB model predicted slightly lower $|\Psi|^{2}$ as compared to the CDL model, as the condensed layer suggested by MPB is larger than that by CDL under the same surface charge (Fig. S3). Although both models predict very similar counterion density and potential profile, CDL model is used here for convenience as the condensed layer is introduced more naturally. 


\section{REFERENCES}

(1) Olechowska, K.; Mach, M.; Hạc-Wydro, K.; Wydro, P. Studies on the Interactions of 2-Hydroxyoleic Acid with Monolayers and Bilayers Containing Cationic Lipid: Searching for the Formulations for More Efficient

Drug Delivery to Cancer Cells. Langmuir 2019, $35 \quad$ (27), 9084-9092. https://doi.org/10.1021/acs.langmuir.9b01326.

(2) Wang, L.; Sun, N.; Wang, Z.; Han, H.; Yang, Y.; Liu, R.; Hu, Y.; Tang, H.; Sun, W. Self-Assembly of Mixed Dodecylamine-Dodecanol Molecules at the Air/Water Interface Based on Large-Scale Molecular Dynamics. J. Mol. Liq. 2019, 276, 867-874. https://doi.org/https://doi.org/10.1016/j.molliq.2018.12.076.

(3) Borukhov, I.; Andelman, D.; Orland, H. Steric Effects in Electrolytes: A Modified Poisson-Boltzmann Equation. Phys. Rev. Lett. 1997, 79 (3), 435-438. https://doi.org/10.1103/PhysRevLett.79.435. 